\section{BMJ Open Respiratory Research}

\title{
Age-stratified burden of pneumococcal community acquired pneumonia in hospitalised Canadian adults from 2010 to 2015
}

Jason LeBlanc, ${ }^{1,2}$ May EISherif,, ${ }^{1,2}$ Lingyun Ye, ${ }^{1,2}$ Donna MacKinnon-Cameron, ${ }^{1,2}$ Ardith Ambrose, ${ }^{1,2}$ Todd F Hatchette, ${ }^{1,2}$ Amanda LS Lang, ${ }^{1,2}$ Hayley D Gillis, ${ }^{1,2}$ Irene Martin, ${ }^{3}$ Walter H Demczuk, ${ }^{3}$ Craig LaFerriere, ${ }^{4}$ Melissa K Andrew, ${ }^{1,2}$ Guy Boivin, ${ }^{5}$ William Bowie, ${ }^{6}$ Karen Green, ${ }^{7}$ Jennie Johnstone, ${ }^{8}$ Mark Loeb, ${ }^{9}$ Anne McCarthy, ${ }^{10}$ Allison McGeer, ${ }^{7}$ Makeda Semret, ${ }^{11}$ Sylvie Trottier, ${ }^{5}$ Louis Valiquette, ${ }^{12}$ Duncan Webster, ${ }^{13}$ Shelly A McNeil ${ }^{1,2}$

\section{ABSTRACT}

Background In Canada, 13-valent pneumococcal conjugate vaccine (PCV13) is recommended in childhood, in individuals at high risk of invasive pneumococcal disease (IPD) and in healthy adults aged $\geq 65$ years for protection against vaccinetype IPD and pneumococcal community-acquired pneumonia (pCAP). Since vaccine recommendations in Canada include both age-based and risk-based guidance, this study aimed to describe the burden of vaccine-preventable PCAP in hospitalised adults by age.

Methods Surveillance for community-acquired pneumonia (CAP) in hospitalised adults was performed prospectively from 2010 to 2015. CAP was radiologically confirmed, and pCAP was identified using blood and sputum culture and urine antigen testing. Patient demographics and outcomes were stratified by age (16-49, 50-64, $\geq 65$ and $\geq 50$ years). Results Of 6666/8802 CAP cases tested, 830 (12.5\%) had pCAP, and 418 (6.3\%) were attributed to a PCV13 serotype. Of PCV13 pCAP, $41 \%$ and $74 \%$ were in adults aged $\geq 65$ and $\geq 50$ years, respectively. Compared with non-pCAP controls, pCAP cases aged $\geq 50$ years were more likely to be admitted to intensive care units (ICUs) and to require mechanical ventilation. Older adults with pCAP were less likely to be admitted to ICU or required mechanical ventilation, given their higher mortality and goals of care. Of pCAP deaths, $67 \%$ and $90 \%$ were in the $\geq 65$ and $\geq 50$ age cohorts, respectively.

Conclusions Adults hospitalised with PCAP in the age cohort of 50-64 years contribute significantly to the burden of illness, suggesting that an age-based recommendation for adults aged $\geq 50$ years should be considered in order to optimise the impact of pneumococcal vaccination programmes in Canada.

employer(s)) 2020. Re-use permitted under CC BY-NC. No commercial re-use. See rights and permissions. Published by BMJ.

For numbered affiliations see end of article.

Correspondence to Dr Jason LeBlanc; jason.leblanc@nshealth.ca

\section{INTRODUCTION}

In Canada, Health Canada authorises the use of vaccines following sufficient evidence on quality, safety and efficacy. In turn, the National Advisory Committee on Immunisation (NACI) makes informed recommendations

\section{Key messages}

To help make informed recommendations for adult immunisation, the Serious Outcomes Surveillance Network of the Canadian Immunisation Research Network performed surveillance prospectively with aims to characterise by age the burden of pneumococcal community-acquired pneumonia (pCAP) in hospitalised Canadian adults and the proportion of pCAP that is attributed to 13-valent pneumococcal conjugate vaccine (PCV13) serotypes.

Adults aged $\geq 50$ years accounted for $74 \%$ of pCAP attributed to PCV13 serotypes, compared with $41 \%$ in adults aged $\geq 65$ years for whom a vaccine recommendation already exists.

These data, along with the clinical burden of pCAP in adults aged 50-64 years, argue the value of immunising adults aged $\geq 50$ years.

on the optimal use of vaccines, which helps provincial or territorial ministries of health develop immunisation programmes for children and adults. ${ }^{1}$ To reduce the burden of pneumococcal diseases, NACI has made agebased and risk-based recommendations for two pneumococcal vaccines currently widely used in Canada: the 23-valent pneumococcal polysaccharide vaccine (PPV23) and the 13-valent pneumococcal conjugate vaccine (PCV13). ${ }^{1}$

Streptococcus pneumoniae (or pneumococcus) infections cause high morbidity and mortality worldwide, with significant impact on public health and healthcare resources. ${ }^{2-5}$ Individuals at risk include children aged $\leq 5$ years, adults aged $\geq 65$ years and individuals of any age with underlying medical conditions. ${ }^{15-12}$ The most serious manifestations of pneumococcal infection include invasive pneumococcal 
diseases (IPDs) and community-acquired pneumonia (CAP). S. pneumoniae is the most frequently identified bacterial pathogen in CAP, and CAP is the most common presentation of pneumococcal disease in older adults. ${ }^{7-12}$ Recent studies in hospitalised Canadian adults demonstrated that $14 \%-23 \%$ of CAP cases were attributable to S. pneumoniae, but these data were likely underestimated. ${ }^{7}$ Pneumococcal community-acquired pneumonia (pCAP) cases were more likely than CAP cases testing negative for S. pneumoniae (non-pCAP) to be admitted to the intensive care unit (ICU), to require mechanical ventilation, and had high mortality. ${ }^{78}$ Vaccine-preventable serotypes of $S$. pneumoniae remain predominant in adults hospitalised with pCAP, despite efforts to confer adult protection indirectly through childhood immunisation. ${ }^{813-15}$

Indirect protection of adults against pneumococcal disease can occur through herd immunity, where childhood immunisation reduces colonisation and subsequent transmission to adults. ${ }^{13-15}$ Following childhood immunisation with a 7-valent conjugate vaccine (PCV7) in Canada, IPD caused by PCV7 serotypes declined significantly in vaccinated children, unvaccinated children and adults. ${ }^{1813-15} \mathrm{~A}$ few years later, non-PCV7 serotypes emerged, and PCV7 was replaced by PCV10 in 2009 in some provinces, and by 2010, PCV13 was implemented in all Canadian provinces. ${ }^{1813-15}$ However, despite broader coverage with PCV13 childhood immunisation and use of PPV23 in adults since 1996, a significant proportion of pCAP and IPD in adults are still caused by PCV13 serotypes. ${ }^{5-8}$ These data suggest that unlike PCV7, childhood immunisation with PCV13 has not provided sufficient indirect protection to adults against pCAP and IPD. ${ }^{8}$

Direct adult immunisation with PCV13 has been the subject of recent debate. ${ }^{1-8}$ The CAPITA trial in the Netherlands demonstrated efficacy in healthy adults aged $\geq 65$ years against vaccine-type non-bacteraemic CAP, bacteraemic pCAP and IPD, but it was undertaken in a background of PCV7- based nd PCV10-based childhood immunisation. ${ }^{16}$ At the time, the potential benefits of PCV13 adult immunisation were unclear in Canada and other countries, given the anticipated herd immunity from PCV13 childhood immunisation. ${ }^{178}$ There are economic modelling data to suggest that PCV13 alone, or PCV13 followed by PPV23, is a cost-effective use of healthcare resources ${ }^{2-4}$; however, this is not universally accepted. ${ }^{17}$ Similar to the USA, ${ }^{18}$ PCV13 received a NACI recommendation for the prevention of IPD and pCAP caused by PCV13 serotypes in immune competent adults aged $\geq 65$ years. ${ }^{1}$ However, in Canada, public funding was not endorsed for individuals without additional risks, at the modelled price. ${ }^{1}$ PCV13 is authorised by Health Canada for indications including use in adults aged $\geq 18$ years for the protection against IPD and pneumonia, but to date, PCV13 is only recommended by NACI for use in adults $<65$ years of age if immune compromised. ${ }^{1}$ There iare some data to suggest that PCV13 and PPV23 should be considered in adults younger than 65 who are at increased risk of pneumococcal disease owing to medical, lifestyle or environmental factors, as the risk of CAP in this population might exceed that in the healthy elderly population. ${ }^{19}$ Other countries have recommended PCV13 in adults aged $\geq 50$ years, irrespective of factors putting them at risk of disease. ${ }^{20-22}$ More Canadian data on disease burden by age could inform recommendations for both PCV13 and PPV23.

The Serious Outcomes Surveillance (SOS) Network of the Canadian Immunisation Research Network (CIRN) recently described the burden of PCAP and IPD in hospitalised adults, and described changes in S. pneumoniae serotype distribution over years $2010-2015 .{ }^{78}$ This study represents a secondary analysis of the data to further characterise, by age, the burden of pCAP and the proportion of pCAP attributed to PCV13 serotypes.

\section{MATERIALS AND METHODS}

All methods were previously described ${ }^{7-9}$ and remained consistent throughout the study period, with the exception that data were stratified by age.

\section{Study eligibility and case definitions}

Dedicated surveillance monitors reviewed all daily adult ( $\geq 16$ years) admissions to identify patients with an acute respiratory illness who were admitted to hospitals from the community with an admitting diagnosis of influenza, CAP, asthma or acute exacerbation of chronic obstructive pulmonary disease, or who presented with any other respiratory tract infection or symptom. A case was enrolled as CAP if a presentation occurred within 72 hours of admission with a new or evolving pulmonary infiltrate on chest radiograph suggestive of pneumonia, with $\geq 2$ signs or symptoms (temperature $>38^{\circ} \mathrm{C}$, cough, sputum production, shortness of breath, pleuritic chest pain, crackles or consolidation on chest examination). Patients were excluded if they failed to meet the CAP case definition, were admitted but discharged or died prior to consent, refused consent or were admitted from a long-term care facility. A case was defined as pCAP if a CAP case had confirmation of $S$. pneumoniae from urine antigen detection or isolation of $S$. pneumoniae from blood or sputum culture. A case was considered non-pCAP if at least one laboratory test was performed, and no results identified S. pneumoniae.

\section{Specimen collection and laboratory testing}

Specimens recovered from participating sites were stored at $-80^{\circ} \mathrm{C}$ and shipped on dry ice to the CIRN SOS Network central laboratory (Halifax, Nova Scotia) for testing. S. pneumoniae isolates were subjected to PCR-based detection and serotyping, ${ }^{78}$ and Quellung reaction was performed at the National Microbiology Laboratory (Winnipeg, Manitoba) using commercial antisera (Statens Serum Institute Diagnostica, Copenhagen, Denmark). Urine was stabilised with $25 \mathrm{mM}$ PIPES buffer ( $\mathrm{pH}$ 6.8) and tested using the pan-pneumococcal Binax NOW S. pneumoniae 
Urinary Antigen Test by the CIRN SOS central laboratory, and using a PCV13 serotype-specific urine antigen detection (ssUAD) assay on a Luminex V.2.0 instrument at Pfizer's Vaccines Research and Development Laboratory (Pearl River, New York). ${ }^{23-25}$

\section{Estimated incidence rates for pCAP}

Specific catchment areas were not defined. Incidence rates for pCAP were estimated by applying the proportion of hospitalised CAP attributed to S. pneumoniae (through prospective surveillance by the CIRN SOS Network) to incidence rates for CAP in hospitalised adults obtained from Discharge Abstract Database data of the Canadian Institute for Health Information (CIHI) (https:// www.cihi.ca/en). Hospitalisations due to all-cause pneumonia by year and age were provided as anonymous and aggregate by CIHI using J12-J18 codes from the International Classification of Diseases, 10th Revision, Canadian Enhancements. Incidence rates were expressed per 100000 persons per year, with $95 \%$ CI. $^{9}$

\section{Statistical analyses}

A regression analysis were used to compare trends over time for proportions or incidences rates, and a $\chi^{2}$ and Fisher's exact test was used to compare two variables. For patient demographics and outcomes in each age category, univariate analyses were performed and multivariate regression analyses were used to identify risk factors for serious outcomes. t-Test and analysis of variance were used for comparing continuous variables, and $\chi^{2}$ was used to compare categorical variables. A $p$ value of $\leq 0.05$ was considered statistically significant.

\section{RESULTS}

Proportion of CAP attributed to $S$. pneumoniae by age

Of 8802 CAP cases enrolled, 2521 (28.6\%) had one diagnostic test for S. pneumoniae, $2606(29.6 \%)$ had two tests, $6666(75.6 \%)$ had at least one test, and $1198(13.6 \%)$ had all tests performed (online supplementary table S1). There was a decline with age in the proportions of CAP cases tested for S. pneumoniae (figure 1A) and S. pneumoniae positivity in CAP (figure 1B), regardless of laboratory test(s) used (online supplementary table $\mathrm{S} 1$ ).

The decline was significant for CAP cases tested using ssUAD, blood culture, sputum culture, at least one (any) test or all tests (with $\mathrm{p}$ values of $0.004,0.033,0.013,0.002$ and 0.021 , respectively). The decline with age for the proportion of CAP cases identified as PCAP was also significant for each laboratory test or combination ( $p$ values of $<0.001,0.016,0.004,<0.001$ and 0.016 , respectively). Of the overall pCAP cases throughout the study period, $22.0 \%, 30.8 \%, 47.1 \%$ and $78.0 \%$ were detected in adults in age groups of $16-49,50-64, \geq 65$ and $\geq 50$ years, respectively (figure $2 \mathrm{~A}$ and online supplementary table S2). No significant differences were noted in proportions whether any or all tests were performed, or between study years (figure 2A,B, and online supplementary table S2).

\section{Incidence rates of pCAP by age}

Whether any or all diagnostic tests for $S$. pneumoniae were performed, incidence rates of pCAP increased with age in each of the study years (figure $2 \mathrm{C}, \mathrm{D}$, and online supplementary tables S3 and S4). The incidence rates in any age group were highest in the early years in the study (2010-2013), the lowest in year 2014 and increased again in 2015 (figure 2C,D, and online supplementary tables S3 and S4). For pCAP cases in whom any test was performed, the decline from 2011 to 2014 was significant for adults aged $16-49$ years $(\mathrm{p}=0.020)$. In addition, a significant rise in pCAP incidence rates was noted from year 2014 to 2015 in adults aged $50-64$ years $(p=0.017)$. For pCAP cases in whom all tests were performed, there was a significant decline in incidence for adults aged 50-64 over years 2012-2014 ( $\mathrm{p}=0.040)$, and a significant rise in incidence from 2014 to 2015 for adults aged $\geq 65$ years $(\mathrm{p}=0.022)$ and all ages $(\mathrm{p}=0.046)$.
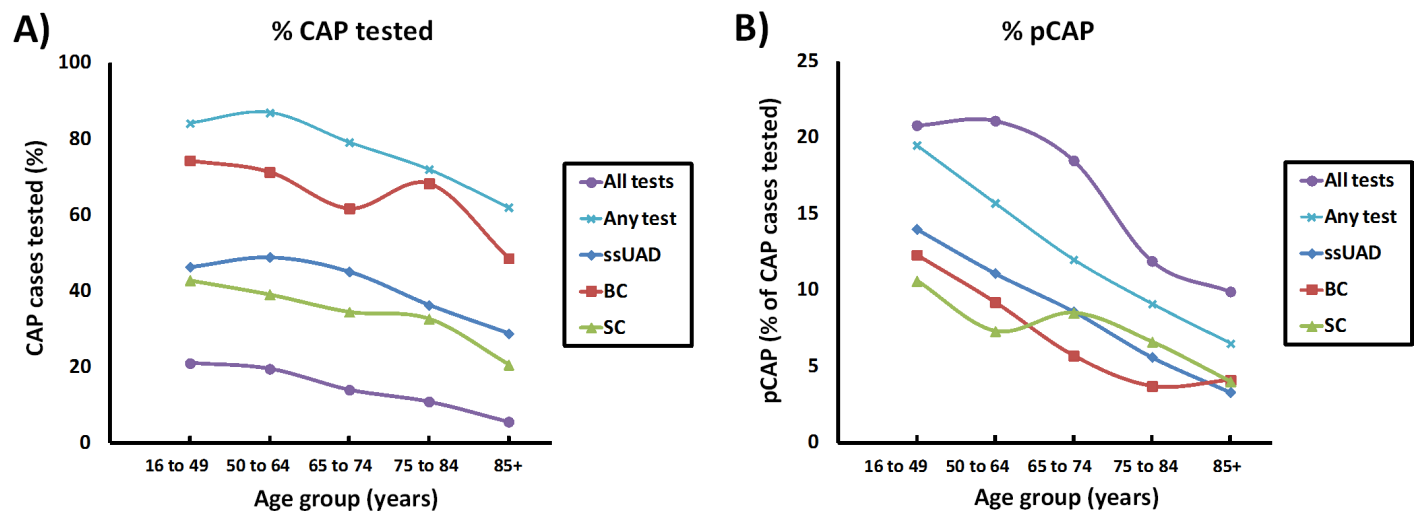

Figure 1 Laboratory testing for Streptococcus pneumoniae in cap cases and proportion of cap identified as pCAP by age. (A) Proportion by age of cap cases tested for S. pneumoniae using SSUAD, BC, SC, at least one (any) diagnostic test or all diagnostic tests. (B) Proportion by age of cap cases identified as pCAP for each laboratory method or testing combinations. $\mathrm{BC}$, blood culture; CAP, cCommunity-acquired pneumonia; pCAP, pneumococcal community-acquired pneumonia; SC, sputum culture; ssUAD, serotype-specific urine antigen testing. 

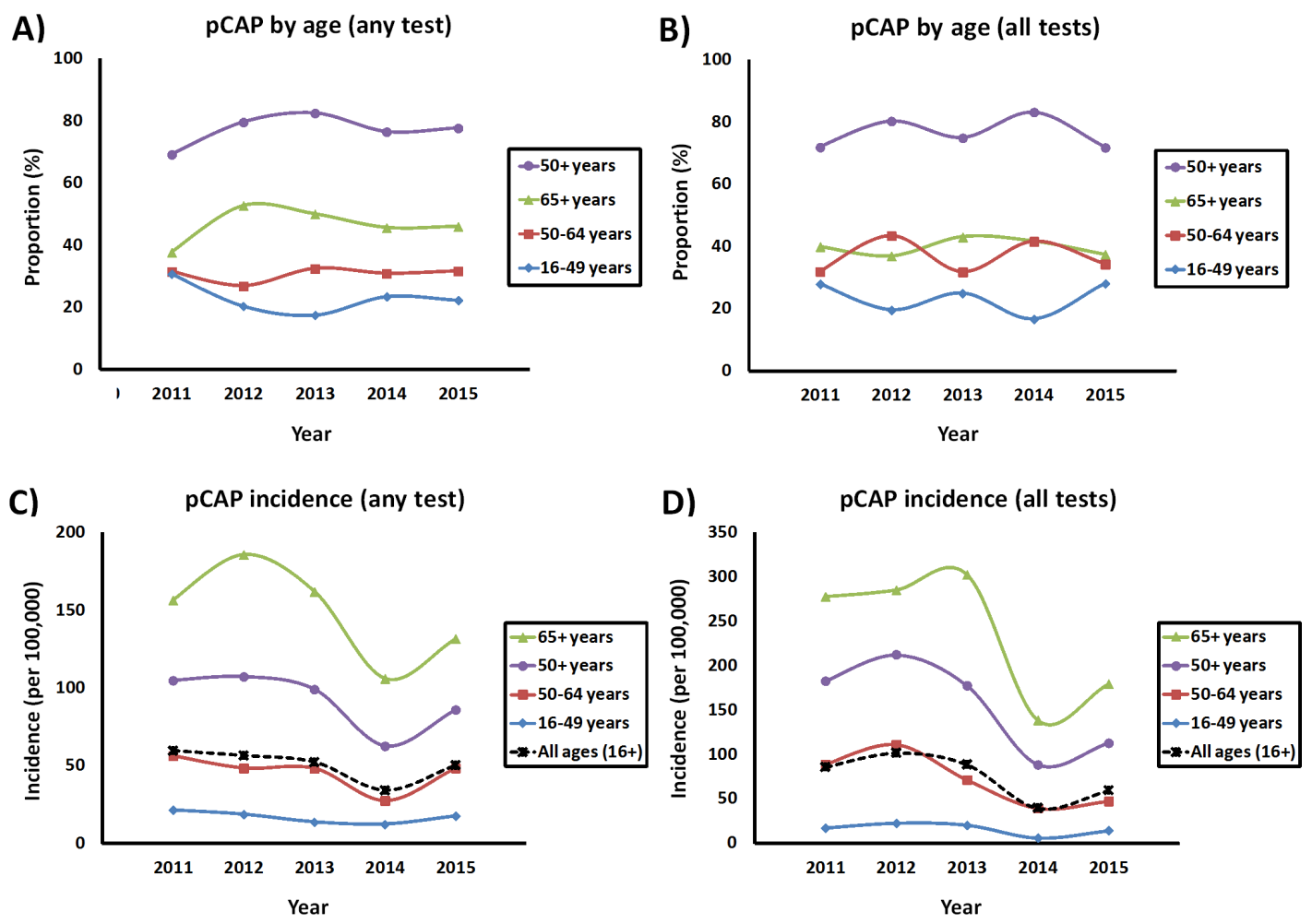

Figure 2 Characterisation of pCAP by age and year. Proportion by age represents pCAP cases identified by (A) any or (B) all diagnostic tests for Streptococcus pneumoniae, (C) incidence rates for pCAP for cases identified by any test and (D) incidence rates for pCAP for cases identified by all tests. pCAP, pneumococcal community-acquired pneumonia.

\section{Proportion of pCAP attributed to PCV13}

No significant changes were reported during the study period for PCV7, PPV23 (non-PCV13) or non-vaccine serotypes $^{8}$; therefore, only pCAP cases attributed PCV13 serotypes were further characterised by age. As seen with pCAP, the proportion of pCAP attributed to PCV13 serotypes was $25.6 \%, 33.0 \%, 41.4 \%$ and $74.4 \%$ in adults aged $16-49,50-64, \geq 65$ and $\geq 50$ years, respectively (figure $3 \mathrm{~A}$ and online supplementary table S5). Similar proportions were observed for patients tested using all laboratory tests for $S$. pneumoniae (figure 3B and online supplementary table S5). No significant differences in proportions were noticed throughout the study period.

\section{Incidence rates of PCV13 pCAP by age}

As seen with pCAP, whether any or all diagnostic tests for S. pneumoniae were performed, incidence rates of pCAP attributed to PCV13 increased with age (figure 3C,D, and online supplementary table S6). Incidence rates of PCV13 pCAP were highest in the early years in the study (2010-2013), declined in 2014 and increased again in 2015 (figure 3C,D, and online supplementary table S6). For pCAP cases in whom any test was performed, the decline in PCV13 serotypes from 2012 to 2014 was significant for adults aged $50-64$ years $(\mathrm{p}=0.032)$ and adults of all ages $(\mathrm{p}=0.014)$. In addition, a significant rise in incidence rates was noted from years 2014 to 2015 in adults aged $50-64, \geq 65$ and $\geq 50$ years $(\mathrm{p}=0.019,0.022$ and 0.018 , respectively). For pCAP cases in whom all tests were performed, there was a significant decline in incidence for adults aged $\geq 50$ years from 2012 to 2014 ( $\mathrm{p}=0.005)$, and a significant rise in incidence from 2014 to 2015 for adults aged $\geq 65$ years $(\mathrm{p}<0.001)$.

\section{S. pneumoniae serotype distribution by age}

No differences in the serotype distribution were noted between age cohorts, with each group demonstrating a predominance for serotypes $3,7 \mathrm{~F}$ and $19 \mathrm{~A}$ (figure $4 \mathrm{~A}$ ). For each serotype, the $\geq 65$ age group accounted for less than half the cases, while the 50-64 age groups represented between $25 \%$ and $35 \%$ of cases (figure $4 \mathrm{~A}$ and online supplementary table S7). Regression analyses were performed for incidence rates over time for the three most predominant serotypes $(3,19 \mathrm{~A}$ and $7 \mathrm{~F})$ (figure 4B-D). A decline over time in incidence rates was seen for serotypes 7F (figure 4B) and 19A, but significant declines were only noted for serotype $7 \mathrm{~F}$ from 2012 to 2014 in adults aged $\geq 65$ years $(\mathrm{p}=0.048), \geq 50$ years $(\mathrm{p}=0.002)$ and all ages $(\mathrm{p}=0.049)$ (figure $4 \mathrm{C}$ and online supplementary table $\mathrm{S} 7$ ). For serotype 3 , there as a significant decline in incidence from 2012 to 2014 for adults aged $\geq 65$ years $(p=0.018)$; however, incidence rates increased from 2014 to 2015 in both adults aged $\geq 65$ and $\geq 50$ years $(\mathrm{p}<0.001$ and 0.011 , respectively).

\section{Patient demographics and outcomes}

As reported previously for all ages, ${ }^{78}$ the mean age of pCAP cases in adults aged $\geq 65$ and $\geq 50$ years was significantly less than that of non-pCAP (table 1). Most patients in any of the age groups had underlying medical comorbidities, but the proportions increased with age for both non-pCAP and pCAP. No significant difference were 

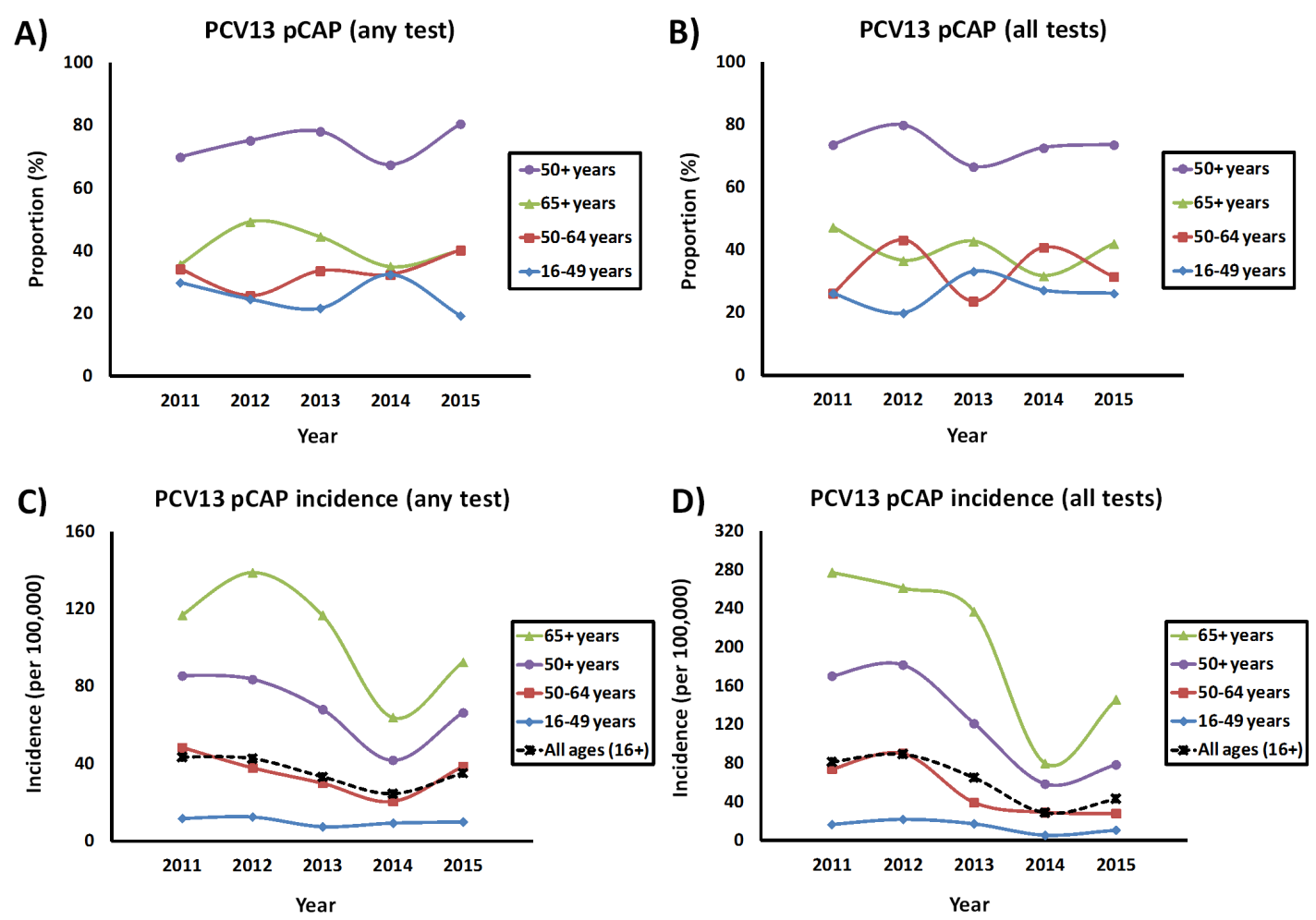

Figure 3 Contribution by age and year of pCAP attributed to PCV13 serotypes. Proportion of cap cases attributed to PCV13 serotypes in cap cases tested using (A) any or (B) all diagnostic tests for Streptococcus pneumoniae, (C) incidence rates for pCAP attributed to PCV13 serotypes for PCAP cases identified by any test and (D) incidence rates for pCAP for cases identified by all tests. pCAP, pneumococcal community-acquired pneumonia.

A)
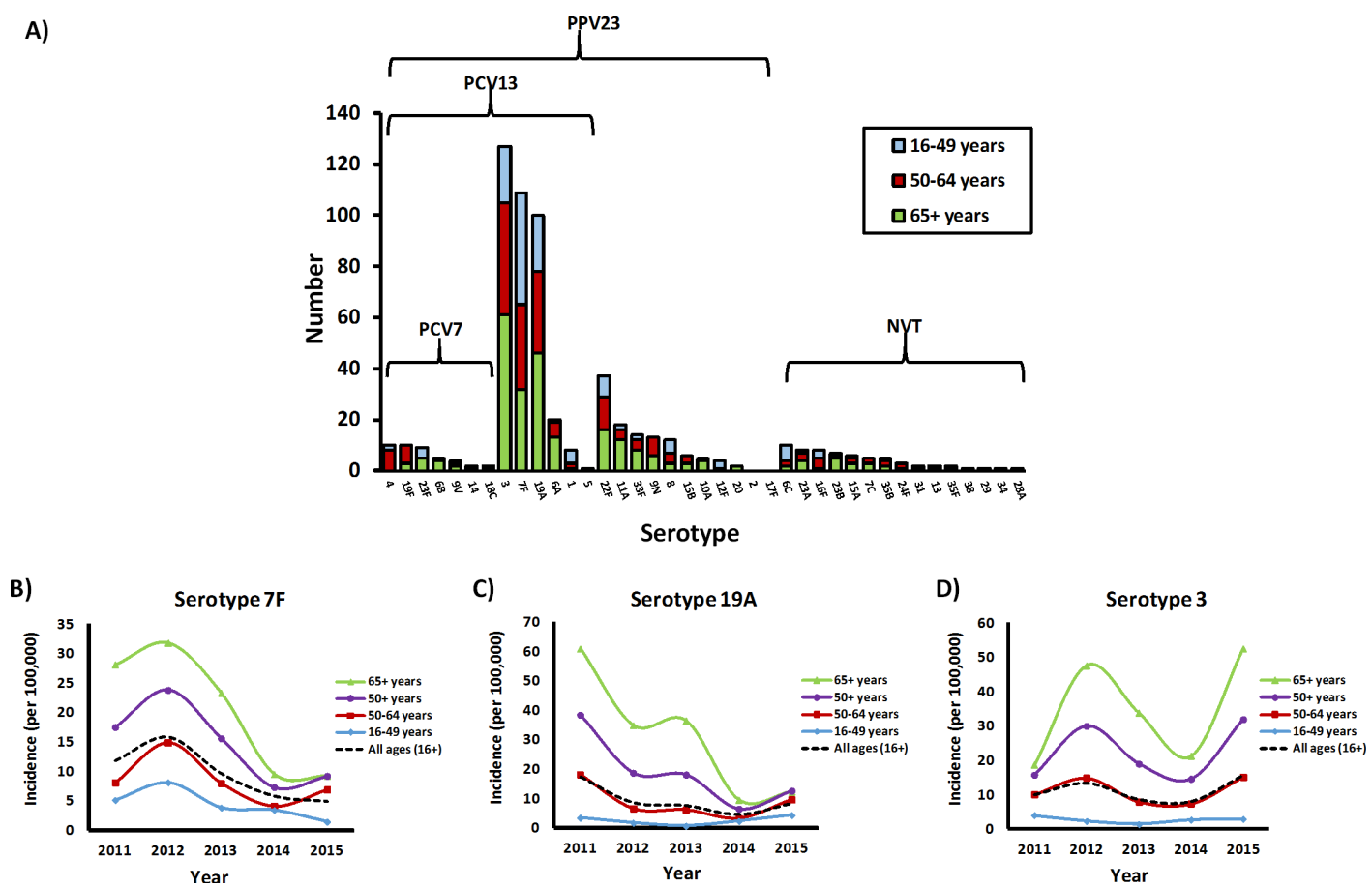

Figure 4 Streptococcus pneumoniae serotype distribution in adults hospitalised with pCAP. (A) Characterisation by age of S. pneumoniae serotypes identified in pCAP $(n=418)$. Incidence rates for the predominant three serotypes are presented over time: (B) serotype 7F, (C) serotype 19A and (D) serotype 3. pCAP, pneumococcal community-acquired pneumonia. 


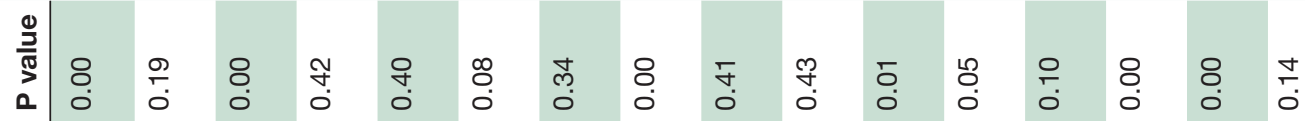

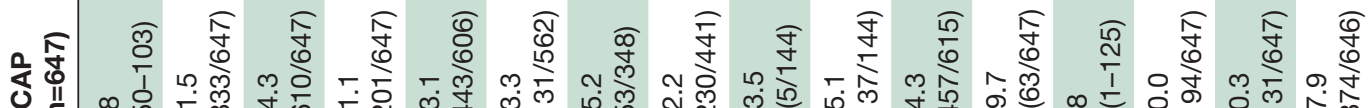

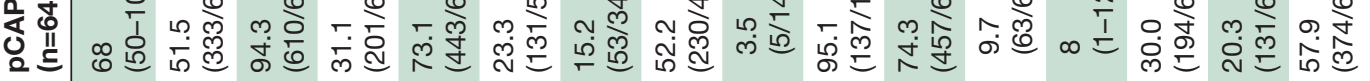

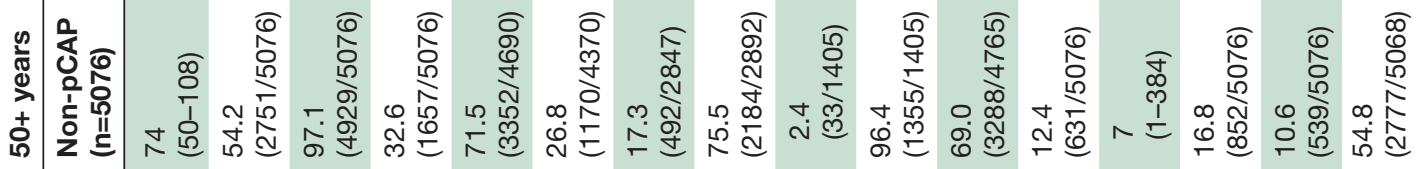

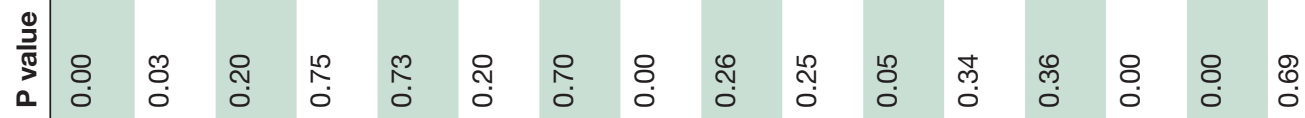

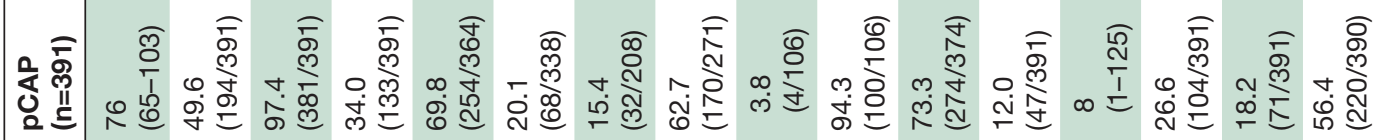

$\stackrel{2}{\circ}$

离

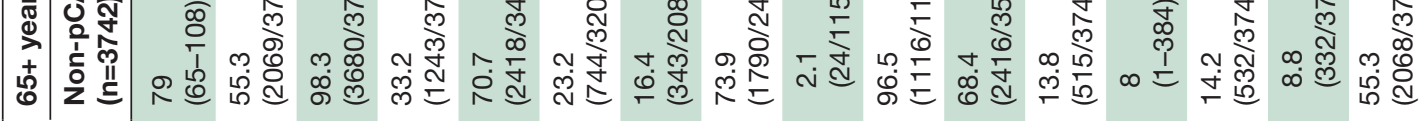

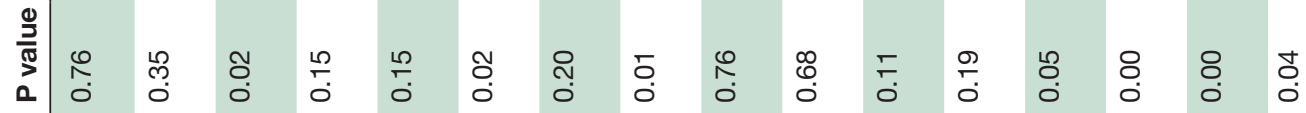

幽空

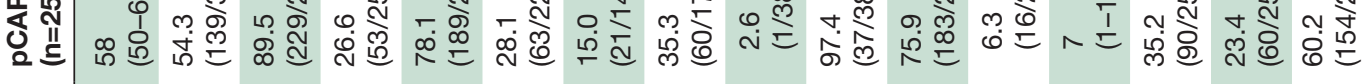

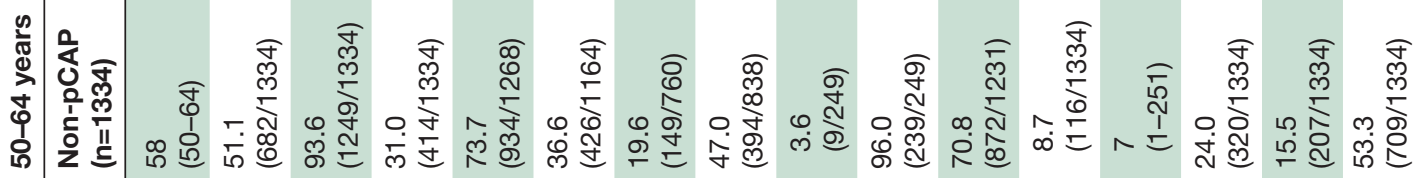

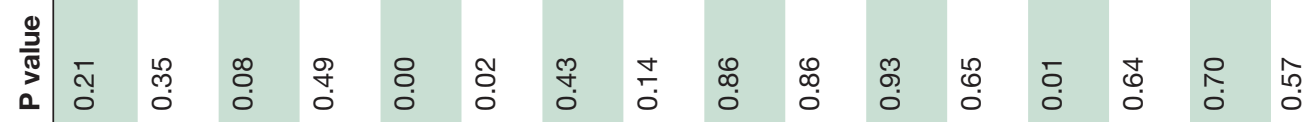

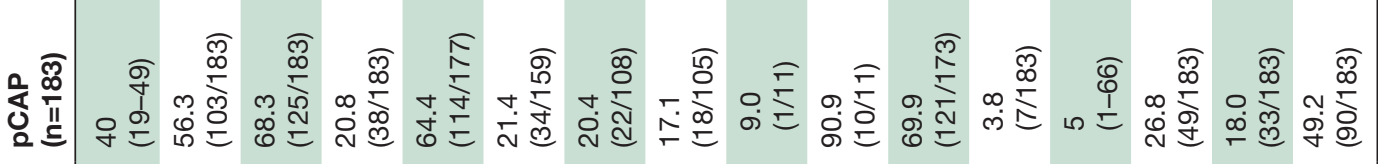

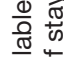


noted in the proportion of patients without pCAP and with pCAP who were immune compromised, but the proportion of individuals who had immune compromising conditions increased with age for both. No differences between non-pCAP and pCAP were noted for concomitant influenza infection. In adults aged 16-49 years, non-pCAP cases were more often obese (body mass index $\geq 30$ ), while smoking was more prominent in the pCAP group. Compared with non-pCAP cases, prior pneumococcal vaccine was less common in pCAP cases in the $\geq 50$-year cohorts. Of the non-pCAP and pCAP cases where immunisation was documented, PPV23 was predominantly used (between $90.9 \%$ and $97.4 \%$, depending on the age cohort). Receipt of antibiotics within 4 hours was more common in pCAP cases for adults aged $\geq 50$ years. In terms of outcomes, mortality (at 30 days) and length of stay increased with age for both non-pCAP and pCAP. For adults aged 16-49, 50-64 and 65 + years, mortality for pCAP was 3.8\%, 6.3\% and $12.0 \%$, respectively. Of 70 pCAP deaths attributed to pCAP, 7 $(10.0 \%), 16(22.8 \%), 47(67.1 \%)$ and $63(90.0 \%)$ were in the age cohorts of $16-49,50-64, \geq 65$ and $\geq 50$ years, respectively. Compared with non-pCAP cases, more ICU admissions and requirement for mechanical ventilation were observed in pCAP cases in all age groups, and these serious outcomes increased with age. Of note, no significant differences in patient demographics were noted between pCAP cases tested by any or all tests for $S$. pneumoniae, with the exception of antibiotics within 4 hours $(\mathrm{p}=0.001)$ (online supplementary table S8). In contrast, pCAP cases identified using all tests were more likely to have been admitted to ICU $(\mathrm{p}=0.000)$ and to require mechanical ventilation $(\mathrm{p}=0.000)$ (online supplementary table S8).

\section{Risk factors for serious outcomes or death}

For non-pCAP cases, increasing age $(\mathrm{p}<0.001)$, male gender $(\mathrm{p}<0.001)$ and compromised immune status $(p<0.001)$ were significant predictors of 30-day mortality (online supplementary table S9). The models were not powered to identify risk factors for death for pCAP. The risk of ICU admission or need for mechanical ventilation decreased with age for non-pCAP and pCAP cases. Receipt of antibiotics within 4 hours of admission was used as proxy of severity on admission rather than a risk factor, but was included in the model. ${ }^{78}$

\section{DISCUSSION}

Characterising the burden of pCAP by age, as well as identifying populations at risk, is important to inform recommendations on pneumococcal vaccines. In Canada, PPV23 has been recommended for adults aged $\geq 65$ years and funded in most provinces since the mid-1990s, and PCV13 was recommended in 2016 for individual use in adults aged $\geq 65$ years, but remains unfunded. ${ }^{1}$ In this study, pCAP cases and pCAP cases attributed to PCV13 serotypes were stratified by age, given there is interest and use of PCV13 in adults aged $\geq 50$ years in other countries. $^{20-22}$ 26-30 These data could also inform age-based PPV23 recommendations in Canada (as all PCV13 serotypes except $6 \mathrm{~A}$ are also covered by PPV23). This study presents several lines of evidence that adults 50-64years of age contribute significantly to the burden of pCAP and argues the value of immunising healthy adults aged $\geq 50$ years.

First, the economic impact of CAP is primarily driven by hospitalisations and deaths. ${ }^{4}$ Adults aged $\geq 65$ years represented only $67 \%$ of deaths attributed to pCAP, $43 \%$ of the ICU admissions and $43 \%$ of patients requiring mechanical ventilation (table 1). Adults 50-64 years contributed significantly to the burden of pCAP disease with $30.8 \%$ of pCAP hospitalisations, $22.8 \%$ of pCAP deaths, $37.0 \%$ of pCAP cases requiring ICU admission and $36.6 \%$ of those requiring mechanical ventilation. Adults aged 16-49 years represented $10.0 \%$ of pCAP deaths, $20.2 \%$ of ICU admissions and $20.1 \%$ of pCAP cases requiring mechanical ventilation. Second, of the pCAP cases identified (figure 2 and online supplementary table S2) and pCAP cases attributed to PCV13 serotypes (figure 3 and online supplementary table S5), adults aged $\geq 65$ years represent less than half $(47.1 \%$ and $41.4 \%$, respectively). Adults aged 50-64 years represented $30.8 \%$ of pCAP and $33.0 \%$ of PCV13 pCAP. Adults aged 16-49 years represented $22.0 \%$ and $25.6 \%$, respectively. Finally, incidence rates for pCAP and pCAP attributed to PCV13 serotypes were highest in adults aged $\geq 65$ years (figures 2 and 3, and online supplementary tables S3, S4 and S6). The incidence rates for adults aged 16-49 years were the lowest, at approximately 10 -fold less than adults aged $\geq 65$ years for any of the study years. With the low incidence rates in adults aged 16-49 years and their smaller contributions to the burden of illness, a recommendation of pneumococcal vaccines for all immune competent adults seems unjustified. However, the observed high incidence rates and burden of illness in adults aged 50-64 years suggests that expanding the age-based recommendation for pneumococcal vaccination to include adults aged 50-64 years would provide optimal protection. Over $90 \%$ adults 50-64 years had at least one comorbidity (table 1) placing them at risk for pneumococcal disease $\mathrm{e}^{2731-34}$ and would therefore be recommended to receive PPV23 under current immunisation programmes, yet only $45 \%$ of adults hospitalised with CAP in this age group had been vaccinated. Similarly, 27\% of hospitalised adults aged 50-64 years were immunocompromised and therefore would be recommended to receive both PCV13 and PPV23 in current Canadian guidelines. ${ }^{1}$ Despite this, only $3.5 \%$ of adults had received PCV13 (table 1). Shifting from riskbased to age-based immunisation recommendations in adults aged 50-64years may facilitate improved uptake in both healthy adults and adults with comorbidities, putting them at higher risk for pnuemococcal disease. ${ }^{31}{ }^{32}$ In Spain, adults aged 60-64 years with medical comorbidities were almost twice as likely to receive influenza vaccine in a region in which the age-based recommendation for 
influenza vaccination was expanded to include adults aged 60 years and older, compared with other regions of the country in which age-based recommendations began at age 65 years and younger adults were recommended to receive vaccine based on risk due to presence of comorbidities. ${ }^{31}$ Similarly, introduction of a universal influenza immunisation programme in the Canadian province of Ontario in 2000 resulted in a more than doubling of the immunisation coverage rate (from $18 \%$ in the period of targeted vaccine recommendations to $42 \%$ following implementation of a universal programme for all Ontarians older than 6 months). ${ }^{32}$ Notably, the largest increases in coverage were observed in adults aged 50-64 years and in people with underlying comorbidities. ${ }^{32}$ Overall, expansion of pneumococcal immunisation recommendations in Canada to include adults aged $\geq 50$ years would capture $78.0 \%$ of pCAP cases, $74.4 \%$ of pCAP attributed to PCV13 serotypes, $90 \%$ of pCAP deaths, $79.8 \%$ of pCAP ICU admissions, $79.9 \%$ of pCAP cases requiring mechanical ventilation, and may be associated with improved vaccine coverage rates among high-risk adults.

In this study, the incidence rates for pCAP in hospitalised adults varied by year and increased with age, which is consistent with trends described for pCAP and IPD in other countries. ${ }^{5} 615212930$ Comparing pCAP incidence rates between countries is challenging, given differences in methodologies, the timing and impact of pneumococcal immunisation programmes, or differences in the patient population; however, it is of interest that incidence rates for $\mathrm{PCAP}$ in this study were much higher than previously recognised in hospitalised Canadian adults. ${ }^{49}$ The prior Canadian studies ${ }^{49}$ used S. pneumoniae-specific International Classification of Diseases, 10th Revision (ICD-10) codes to capture pCAP, retrospectively, but these codes only capture a small subset of cases when testing occurred as standard of care because the use of empiric antibiotics obviates the need to pursue the aetiology of CAP in most cases. In this study, validated ICD-10 codes $^{33}$ for adult hospitalisation with CAP were used to provide a denominator for incidence rates, and the contribution of pCAP to CAP was assessed with prospective disease surveillance. ${ }^{78}$ The prospective identification of CAP cases and active engagement of study staff with clinicians to encourage collection of blood and sputum cultures, as well as urine for antigen detection among both bacteraemic and non-bacteraemic pCAP cases in this study provided a much more complete assessment of the contribution of S. pneumoniae to hospitalised CAP than would typically be achieved through standard care. ${ }^{78}$ The benefits of the PCV13 ssUAD was particularly evident, as it identified many PCV13 pCAP cases that would otherwise have been missed by IPD surveillance. ${ }^{7} 810-1219$ 23-27 While PCV13 ssUAD had benefits, the contributions of non-PCV13 serotypes and non-vaccine serotypes were under-represented, as they were only identified from blood or sputum culture. A 24-valent ssUAD was recently described that can also detect PPV23 (non-PCV13) serotypes, ${ }^{29}$ but it was not available at the time of this study.
While pCAP incidence rates were higher than previously recognised in Canada, the burden of pCAP likely remains underestimated. Only $14 \%$ of CAP patients had all diagnostic tests for S. pneumoniae performed, and $76 \%$ had at least one test (online supplementary table $\mathrm{S} 1)$. Due to the incomplete testing, a proportion of pCAP cases in all age cohorts would have been misclassified as non-pCAP, and this would underestimate pCAP disease. This may be most marked in the oldest age groups as a decline in testing for $S$. pneumoniae with age was observed with a resultant reduction in the proportion of CAP attributed to PCAP as age increased (figure 1). Reasons for decreased testing with age are likely multifactorial. It is possible that goals of care in older adults with CAP would less likely include aggressive pursuit of an aetiological diagnosis of CAP. ${ }^{34}$ Higher mortality in older adults may have precluded testing for S. pneumoniae (table 1). ${ }^{921} 272934-37$ Alternatively, with comorbidities or immune compromising conditions increasing with age (table 1), older adults might have a lower threshold for hospitalisation and may not require as extensive laboratory investigations for patient management. ${ }^{34} 37$ Regardless, the methods used in this study were consistent throughout the study years. These limitations, among others (ie, missing demographic data in some patients), are similar to those of other studies of pneumococcal disease epidemiology. ${ }^{38}$

The strengths of this study include its prospective design, with specimen and data collection performed in several adult hospitals across five provinces. The timing spanned a 5-year period following introduction of PCV13 into Canadian childhood immunisation programmes, which were implemented in all provinces by 2010. In this period, herd effects were anticipated in adults. ${ }^{178}$ Consistent with the previously reported proportion of PCV13 serotypes over time in hospitalised adults, ${ }^{8}$ this study showed a significant decline in the incidence rates of serotypes $7 \mathrm{~F}$ and 19A, along with the persistence of serotype 3 (figure 4). Similar trends were seen in all age cohorts and are consistent with national IPD surveillance ${ }^{56}$ and other recent studies. ${ }^{14} 2939$ Many potential reasons for the persistence of serotype 3 have been proposed. ${ }^{8142940}$ However, there is evidence that direct PCV13 immunisation of healthy adults aged $\geq 65$ years is effective against PCV13 serotypes, including serotype $3 .^{16}{ }^{40}$ It remains to be seen whether direct PCV13 immunisation of adults will help reduce the remaining burden of pCAP in Canadian adults. With lack of provincial funding, PCV13 uptake in adults has been low to date. Recently, both NACI and the Advisory Committee on Immunisation Practices in the USA cited a high economic cost and minimal public health benefit of PCV13 due to the low remaining disease burden of PCV13 serotypes in adults aged $\geq 65$ years. ${ }^{141}$ In more recent years, national IPD surveillance showed further decline in PCV13 serotypes (except serotype 3). ${ }^{5}$ However, this retrospective surveillance focused on IPD would miss many pCAP cases, as the collection of sputum and urine is not standard of care, and blood cultures are 
not consistently performed in all CAP cases. As this study spanned years 2010-2015, ongoing prospective surveillance is required to assess more recent trends in pneumococcal disease.

Overall, this study demonstrated that the burden of pCAP was greater than previously recognised, and a significant proportion of the burden of pCAP was attributed to adults aged 50-64 years. Expansion of age-based pneumococcal vaccine recommendations to include adults aged $\geq 50$ years should be considered to optimise the impacts of pneumococcal vaccination programmes.

\section{Author affiliations}

${ }^{1}$ Canadian Center for Vaccinology (CCfV), Nova Scotia Health Authority, Halifax, Nova Scotia, Canada

${ }^{2}$ Department of Medicine, Dalhousie University, Halifax, Nova Scotia, Canada

${ }^{3}$ Streptocococcus \& STI Unit, National Microbiology Laboratory (NML), Public Health Agency of Canada (PHAC), Winnipeg, Manitoba, Canada

${ }^{4}$ Canvax, Mississauga, Ontario, Canada

${ }^{5}$ Department of Microbiology-Immunology and Infectious Diseases, Centre Hospitalier Universitaire de Québec, Quebec City, Quebec, Canada

${ }^{6}$ Department of Medicine, Vancouver General Hospital and University of British Columbia, Vancouver, British Columbia, Canada

${ }^{7}$ Department of Microbiology, Mount Sinai Hospital, Toronto, Ontario, Canada

${ }^{8}$ Department of Medicine, Public Health Ontario and University of Toronto,

Toronto, Ontario, Canada

${ }^{9}$ Department of Medicine, McMaster University, Hamilton, Ontario, Canada

${ }^{10}$ Division of Infectious Diseases, Ottawa Hospital General Campus and University of Ottawa, Hamilton, Ontario, Canada

${ }^{11}$ Department of Medicine, McGill University Health Centre, Montreal, Quebec, Canada

${ }^{12}$ Department of Microbiology and Infectiology, Centre Intégré Universitaire de Santé et de Services Sociaux de l'Estrie - Centre Hospitalier Universitaire de Sherbrooke, Sherbrooke, Québec, Canada

${ }^{13}$ Department of Medicine, Saint John Regional Hospital, Saint John, New Brunswick, Canada

Acknowledgements The authors thank all Canadian Immunization Research Network Serious Outcomes Surveillance surveillance monitors, as well as staff at the Canadian Center for Vaccinology, who were instrumental in the recruitment, collection and processing of community-acquired pneumonia and invasive pneumococcal disease specimens. Also, the technical assistance of the laboratory staff at the Streptococcus and Sexual Transmitted Diseases Unit of the National Microbiology Laboratory, Public Health Agency of Canada, was greatly appreciated.

Contributors The Canadian Immunization Research Network (CIRN) Serious Outcomes Surveillance (SOS) principal investigator SAMcN and CIRN SOS site investigators AMcC, AMcG, DW, GB, JJ, JLB, KG, LV, MKA, ML, MS, ST and WB were involved in the conception and design of the study; SAMcN, JLB, TFH and ME conducted/supervised the CIRN SOS Network central laboratory; SAMCN, ME and AA were involved in CIRN SOS site coordination. DM-C, LY and JL were involved in data management and statistical support. JLB, ALSL, HDG, WHD and IM were involved in method development and molecular testing for the study. IM provided reference serological testing at the National Microbiology Laboratory. JL drafted the manuscript and interpreted the data. All authors revised the manuscript and provided important intellectual content, and agreed on the final version submitted for publication.

Funding This work was supported by the Public Health Agency of Canada the Canadian Institutes of Health Research and by an investigator-initiated research grant to Canadian Immunization Research Network from Pfizer Canada. Serotype-specific urine antigen detection was performed by Pfizer's Vaccines Research and Development Laboratory (Pearl River, New York, USA). No study sponsors were involved in study design, specimen collection, analysis and interpretation of data, scientific writing or decision to submit the paper for publication

Competing interests SAM received research grants and personal fees from GlaxoSmithKline, Pfizer and Sanofi Pasteur and personal fees from Merck; JL and TH participated on investigator-initiated grants sponsored by GSK and Pfizer; JL received grants from Merck; LV received research grants from GlaxoSmithKline, Pfizer, Optimer, Cubist and Merck, and personal fees from Merck, Optimer and
Cubist. ML received grants from Pfizer. CL was a former employee for Pfizer Canada, Kirkland Québec, Québec. No other conflicts were declared.

Patient consent for publication Not required.

ETHICS This study was approved by the research ethics boards (REB) at each participating hospital, and written informed consent was provided by each participant or their legally authorised representative. REB protocol numbers varied by institution as follows: 2011-279-10-164 for Centre Intégré Universitaire de Santé et de Services Sociaux de I'Estrie-Centre Hospitalier Universitaire de Sherbrooke (Sherbrooke, Québec), 2012-641 and C10-10-097 for Centre Hospitalier Universitaire de Québec (Québec, Québec), 15-052 for Health Sciences North (Sudbury Ontario), 12-268 for McGill University Health Centre (Montréal, Québec), 10-473 for McMaster University (Hamilton, Ontario), 2015-2211 for the Moncton General Hospital (Moncton, New Brunswick), 09-202-E for Mount Sinai Hospital and University of Toronto (Toronto, Ontario), 11-0182 for North York General Hospital (Toronto, Ontario), 103577 for Nova Scotia Health Authority (Halifax, Nova Scotia), 2010537-01 hour for 0ttawa Hospital General Campus and University of Ottawa (Ottawa, Ontario), 2011-1562 for Saint John Regional Hospital (Saint John, New Brunswick), 484-1111-Inf 017 for Toronto East General Hospital (Toronto, Ontario) and V11-02550 for Vancouver General Hospital and University of British Columbia (Vancouver, British Columbia).

Provenance and peer review Not commissioned; externally peer reviewed.

Data availability statement All data relevant to the study are included in the article or uploaded as supplementary information.

Open access This is an open access article distributed in accordance with the Creative Commons Attribution Non Commercial (CC BY-NC 4.0) license, which permits others to distribute, remix, adapt, build upon this work non-commercially, and license their derivative works on different terms, provided the original work is properly cited, appropriate credit is given, any changes made indicated, and the use is non-commercial. See: http://creativecommons.org/licenses/by-nc/4.0/

\section{REFERENCES}

1 National Advisory Committee on immunization (NACl). Available: https://www.canada.ca/en/public-health/services/immunization/ national-advisory-committee-on-immunization-naci.html [Accessed Dec 2019].

2 Weycker D, Sato R, Strutton D, et al. Public health and economic impact of 13-valent pneumococcal conjugate vaccine in US adults aged $\geq 50$ years. Vaccine 2012;30:5437-44.

3 Huang SS, Johnson KM, Ray GT, et al. Healthcare utilization and cost of pneumococcal disease in the United States. Vaccine 2011;29:3398-412.

4 Atwood M, Beausoleil L, Breton M-C, et al. Cost-Effectiveness of alternative strategies for use of 13-valent pneumococcal conjugate vaccine (PCV13) in Canadian adults. Can J Public Health 2018;109:756-68.

5 Public Agency of Canada (PHAC). National laboratory surveillance of invasive streptococcal disease in Canada. annual summary, 2017. Available: https://www.canada.ca/en/public-health/services/ publications/drugs-health-products/national-laboratory-surveillanceinvasive-streptococcal-disease-annual-summary-2017.html [Accessed Dec 2019].

6 Demczuk WHB, Martin I, Desai S, et al. Serotype distribution of invasive Streptococcus pneumoniae in adults 65 years of age and over after the introduction of childhood 13-valent pneumococcal conjugate vaccination programs in Canada, 2010-2016. Vaccine 2018;36:4701-7.

7 LeBlanc JJ, ElSherif M, Ye L, et al. Burden of vaccine-preventable pneumococcal disease in hospitalized adults: a Canadian immunization research network (CIRN) serious outcomes surveillance (SOS) network study. Vaccine 2017;35:3647-54.

8 LeBlanc JJ, ElSherif M, Ye L, et al. Streptococcus pneumoniae serotype 3 is masking PCV13-mediated herd immunity in Canadian adults hospitalized with community acquired pneumonia: a study from the serious outcomes surveillance (SOS) network of the Canadian immunization research network (CIRN). Vaccine 2019;37:5466-73.

9 McNeil SA, Qizilbash N, Ye J, et al. A retrospective study of the clinical burden of hospitalized all-cause and pneumococcal pneumonia in Canada. Can Respir J 2016;2016:1-10.

10 Jain S, Self WH, Wunderink RG, et al. Community-Acquired pneumonia requiring hospitalization among U.S. adults. $N$ Engl $J$ Med 2015;373:415-27.

11 Said MA, Johnson HL, Nonyane BAS, et al. Estimating the burden of pneumococcal pneumonia among adults: a systematic review and meta-analysis of diagnostic techniques. PLoS One 2013;8:e60273. 
12 Rodrigo C, Bewick T, Sheppard C, et al. Impact of infant 13-valent pneumococcal conjugate vaccine on serotypes in adult pneumonia. Eur Respir J 2015;45:1632-41.

13 Centers for Disease Control and Prevention (CDC). Direct and indirect effects of routine vaccination of children with 7-valent pneumococcal conjugate vaccine on incidence of invasive pneumococcal disease - United States, 1998-2003. Morb Mortal Wkly Rep 2005;54:893-7.

14 Isturiz RE, Ramirez J, Self WH, et al. Pneumococcal epidemiology among US adults hospitalized for community-acquired pneumonia. Vaccine 2019;37:3352-61.

15 Kislaya I, Rodrigues AP, Sousa-Uva M, et al. Indirect effect of 7 -valent and 13-valent pneumococcal conjugated vaccines on pneumococcal pneumonia hospitalizations in elderly. PLoS One 2019;14:e0209428.

16 Bonten MJM, Huijts SM, Bolkenbaas M, et al. Polysaccharide conjugate vaccine against pneumococcal pneumonia in adults. $N$ Engl J Med 2015;372:1114-25.

17 van Hoek AJ, Miller E. Cost-Effectiveness of vaccinating immunocompetent $\geq 65$ year olds with the 13-valent pneumococcal conjugate vaccine in England. PLoS One 2016;11:e0149540.

18 Tomczyk S, Bennett NM, Stoecker C, et al. Use of 13-valent pneumococcal conjugate vaccine and 23-valent pneumococcal polysaccharide vaccine among adults aged $\geq 65$ years: recommendations of the Advisory Committee on immunization practices (ACIP). Morb Mortal Wkly Rep 2014;63:822-5.

19 Kaplan A, Arsenault P, Aw B, et al. Vaccine strategies for prevention of community-acquired pneumonia in Canada: who would benefit most from pneumococcal immunization? Can Fam Physician 2019;65:625-33.

20 Sings HL. Pneumococcal conjugate vaccine use in adults - Addressing an unmet medical need for non-bacteremic pneumococcal pneumonia. Vaccine 2017;35:5406-17.

$21 \mathrm{Heo} J Y$, Seo YB, Choi WS, et al. Incidence and case fatality rates of community-acquired pneumonia and pneumococcal diseases among Korean adults: catchment population-based analysis. PLoS One 2018;13:e0194598.

22 Castiglia P. Recommendations for pneumococcal immunization outside routine childhood immunization programs in Western Europe. Adv Ther 2014;31:1011-44.

23 Huijts SM, Pride MW, Vos JMI, et al. Diagnostic accuracy of a serotype-specific antigen test in community-acquired pneumonia. Eur Respir J 2013;42:1283-90.

24 Pride MW, Huijts SM, Wu K, et al. Validation of an immunodiagnostic assay for detection of 13 Streptococcus pneumoniae serotypespecific polysaccharides in human urine. Clin Vaccine Immunol 2012;19:1131-41.

25 Wunderink RG, Self WH, Anderson EJ, et al. Pneumococcal community-acquired pneumonia detected by serotype-specific urinary antigen detection assays. Clin Infect Dis 2018;66:1504-10.

26 Regev-Yochay G, Chowers M, Chazan B, et al. Distribution of 13-Valent pneumococcal conjugate vaccine serotype streptococcus pneumoniae in adults 50 Years and Older presenting with community-acquired pneumonia in Israel. Hum Vaccin Immunother 2018;14:2527-32.

27 Sherwin RL, Gray S, Alexander R, et al. Distribution of 13-valent pneumococcal conjugate vaccine Streptococcus pneumoniae serotypes in US adults aged $\geq 50$ years with community-acquired pneumonia. J Infect Dis 2013;208:1813-20.

28 Wateska AR, Nowalk MP, Lin CJ, et al. Cost-effectiveness of adult pneumococcal vaccination policies in underserved minorities aged 50-64 years compared to the US general population. Vaccine 2019;37:2026-33.

29 Pick H, Daniel P, Rodrigo C, et al. Pneumococcal serotype trends, surveillance and risk factors in UK adult pneumonia, 2013-18. Thorax 2020;75:38-49.

30 Pelton SI, Bornheimer R, Doroff R, et al. Decline in pneumococcal disease attenuated in older adults and those with comorbidities following universal childhood PCV13 immunization. Clin Infect Dis 2019:68:1831-8.

31 Jiménez-García R, Rodríguez-Rieiro C, Hernández-Barrera V, et al. Effectiveness of age-based strategies to increase influenza vaccination coverage among high risk subjects in Madrid (Spain). Vaccine 2011;29:2840-5.

32 Kwong JC, Rosella LC, Johansen $\mathrm{H}$. Trends in influenza vaccination in Canada, 1996/97 to 2005. Health Reports 2007;18:1-11.

33 Skull SA, Andrews RM, Byrnes GB, et al. ICD-10 codes are a valid tool for identification of pneumonia in hospitalized patients aged $>$ or $=65$ years. Epidemiol Infect 2008;136:232-40.

34 Storms AD, Chen J, Jackson LA, et al. Rates and risk factors associated with hospitalization for pneumonia with ICU admission among adults. BMC Pulm Med 2017;17:208.

35 Cillóniz C, Polverino E, Ewig S, et al. Impact of age and comorbidity on cause and outcome in community-acquired pneumonia. Chest 2013;144:999-1007.

36 Torres A, Cillóniz C, Blasi F, et al. Burden of pneumococcal community-acquired pneumonia in adults across Europe: a literature review. Respir Med 2018;137:6-13.

37 Jarrett PG, Rockwood K, Carver D, et al. Illness presentation in elderly patients. Arch Intern Med 1995;155:1060-4.

38 van Werkhoven $\mathrm{CH}$, Huijts SM, Paling FP, et al. The scrutiny of identifying community-acquired pneumonia episodes quantified bias in absolute effect estimation in a population-based pneumococcal vaccination trial. J Clin Epidemiol 2016;69:185-92.

39 Forstner C, Kolditz M, Kesselmeier M, et al. Pneumococcal conjugate serotype distribution and predominating role of serotype 3 in German adults with community-acquired pneumonia. Vaccine 2020;38:1129-36.

40 Gessner BD, Jiang Q, Van Werkhoven $\mathrm{CH}$, et al. A post-hoc analysis of serotype-specific vaccine efficacy of 13-valent pneumococcal conjugate vaccine against clinical community acquired pneumonia from a randomized clinical trial in the Netherlands. Vaccine 2019;37:4147-54.

41 Matanock A, Lee G, Gierke R, et al. Use of 13-valent pneumococcal conjugate vaccine and 23-valent pneumococcal polysaccharide vaccine among adults $\geq 65$ years. MMWR 2019;68:1069-75. 